\title{
The salinity tolerant poplar database (STPD): a comprehensive database for studying tree salt-tolerant adaption and poplar genomics
}

\author{
Yazhen $\mathrm{Ma}^{\dagger}$, Ting Xu', Dongshi Wan ${ }^{\dagger}$, Tao Ma, Sheng Shi, Jianquan Liu and Quanjun Hu*
}

\begin{abstract}
Background: Soil salinity is a significant factor that impairs plant growth and agricultural productivity, and numerous efforts are underway to enhance salt tolerance of economically important plants. Populus species are widely cultivated for diverse uses. Especially, they grow in different habitats, from salty soil to mesophytic environment, and are therefore used as a model genus for elucidating physiological and molecular mechanisms of stress tolerance in woody plants.

Description: The Salinity Tolerant Poplar Database (STPD) is an integrative database for salt-tolerant poplar genome biology. Currently the STPD contains Populus euphratica genome and its related genetic resources. P. euphratica, with a preference of the salty habitats, has become a valuable genetic resource for the exploitation of tolerance characteristics in trees. This database contains curated data including genomic sequence, genes and gene functional information, non-coding RNA sequences, transposable elements, simple sequence repeats and single nucleotide polymorphisms information of P. euphratica, gene expression data between P. euphratica and Populus tomentosa, and whole-genome alignments between Populus trichocarpa, P. euphratica and Salix suchowensis. The STPD provides useful searching and data mining tools, including GBrowse genome browser, BLAST servers and genome alignments viewer, which can be used to browse genome regions, identify similar sequences and visualize genome alignments. Datasets within the STPD can also be downloaded to perform local searches.

Conclusions: A new Salinity Tolerant Poplar Database has been developed to assist studies of salt tolerance in trees and poplar genomics. The database will be continuously updated to incorporate new genome-wide data of related poplar species. This database will serve as an infrastructure for researches on the molecular function of genes, comparative genomics, and evolution in closely related species as well as promote advances in molecular breeding within Populus. The STPD can be accessed at http://me.lzu.edu.cn/stpd/.
\end{abstract}

Keywords: Salt-tolerant, Populus, Genome database, Tree adaptation, Abiotic stress

\section{Background}

Salinity is a main environmental constraint that renders fields unproductive. It is also one of the most severe abiotic stress factors affecting plant growth and agricultural production worldwide [1]. To cope with this intractable problem, many researches have been undertaken to explore the physiological and molecular mechanisms of plants that naturally display high salt resistance or use plant breeding and biotechnological approaches to

\footnotetext{
* Correspondence: huquanjun@gmail.com

'Equal contributors

Molecular Ecology Group, State Key Laboratory of Grassland and

Agro-Ecosystems, School of Life Sciences, Lanzhou University, Lanzhou 730000Gansu, China
}

(c) 2015 Ma et al.; licensee BioMed Central. This is an Open Access article distributed under the terms of the Creative Commons Attribution License (http://creativecommons.org/licenses/by/4.0), which permits unrestricted use, distribution, and reproduction in any medium, provided the original work is properly credited. The Creative Commons Public Domain Dedication waiver (http://creativecommons.org/publicdomain/zero/1.0/) applies to the data made available in this article unless otherwise stated.

enhance the stress resistance of salt-sensitive plant species, especially those with significant economic importance $[2-5]$.

The genus Populus is widely distributed and consists of many species that play important parts in bio-energy production, environmental protection and afforestation on degraded soils [6]. In addition to their conspicuous economic values, these woody species also exhibit different degrees of stress resistance as a consequence of adaptation to different habitats $[7,8]$, thus being very suitable to address tree-specific questions of salt stress tolerance [9].

Populus euphratica Oliv. is a salinity tolerant poplar, which occurs in semiarid and arid areas [10]. It grows 
under unfavorable conditions such as saline soils, but sustains higher photosynthetic and growth rates than other poplar species under high salinity $[3,11]$. With the extraordinary adaptation to salt stress, it has become a model for elucidating salt resistance mechanisms in trees [12]. Breeders have tried to increase tree salt tolerance by crossing $P$. euphratica with other economical species. However, successful hybridization with positive features is scarce [7]. Therefore, molecular breeding provides a promising alternative.

Based on the recently completed whole genome sequence of the $P$. euphratica [12], we have built a comprehensive web-based database, STPD (http://me.lzu. edu.cn/stpd/), to facilitate researches on salinity tolerance and molecular breeding of Populus.

\section{Construction and content}

The STPD currently gives public access to $P$. euphratica genome assembly version 1.1, which was sequenced and assembled using the fosmid pooling and hierarchical approach. The final assembly covers a total length of $496.5 \mathrm{Mb}$ [12], and 34,279 protein-coding genes were predicted in the whole genome. In addition, 764 transfer RNAs, 706 ribosomal RNAs, 4,826 small nuclear RNAs and 266 microRNAs that supported by small RNA sequencing data were identified and included in the database. We also incorporated gene expression data, which is based on time-course profiling of differentially expressed genes between P. euphratica and Populus tomentosa (a salt-sensitive poplar) in response to salt stress. Moreover, a total of 18,938 universal pairs of simple sequence repeat (SSRs) primers were identified in the syntenic regions of P. euphratica and Populus trichocarpa, and these SSRs can be converted into genetic markers across most poplar species. The STPD includes GBrowse genome browser, gene search function, BLAST sequence searching and other intuitive tools to facilitate the analysis of the genetic data in salt-tolerant Populus (Figure 1).

\section{Genome component}

Repeated sequences within the $P$. euphratica genome were identified by RepeatMasker (http://www.repeatmasker. org) with two different libraries. The first one is Repbase TE library (http://www.girinst.org/repbase) while the second one was produced by RepeatModeler, which yielded classification information for each repeat family and consensus sequences as a repeat library.

\section{Gene component}

We obtained the gene dataset of $P$. euphratica using a variety of strategies, including RNA-seq, homology and $a b$ initio gene prediction. The total predicted gene number is 34,279. Among these, 20038 (58.46\%) genes are supported by RNA-seq and 32182 (93.88\%) have a homologue either in Ricinus communis, Cucumis sativus, P. trichocarpa or Prunus persica [12]. This combined gene dataset was then used as a reference and has been integrated into the STPD. The genes were annotated with a variety of databases including Swiss-Prot/TrEMBL (http://www.uniprot.org), KEGG (http://www.genome.jp/kegg), InterPro (http://www. ebi.ac.uk/interpro) and Gene Ontology (http://geneontology. org). Swiss-Prot and TrEMBL annotations for the predicted proteins were generated by performing BLASTP searching (E-value $\leq 10^{-5}$ ) against the Swiss-Prot and TrEMBL databases. Genes were mapped to KEGG pathway using KAAS [13]. We also used InterProScan to annotate domains and motifs by comparing the genes to the public Pfam, PRINTS, PROSITE, ProDom and SMART,

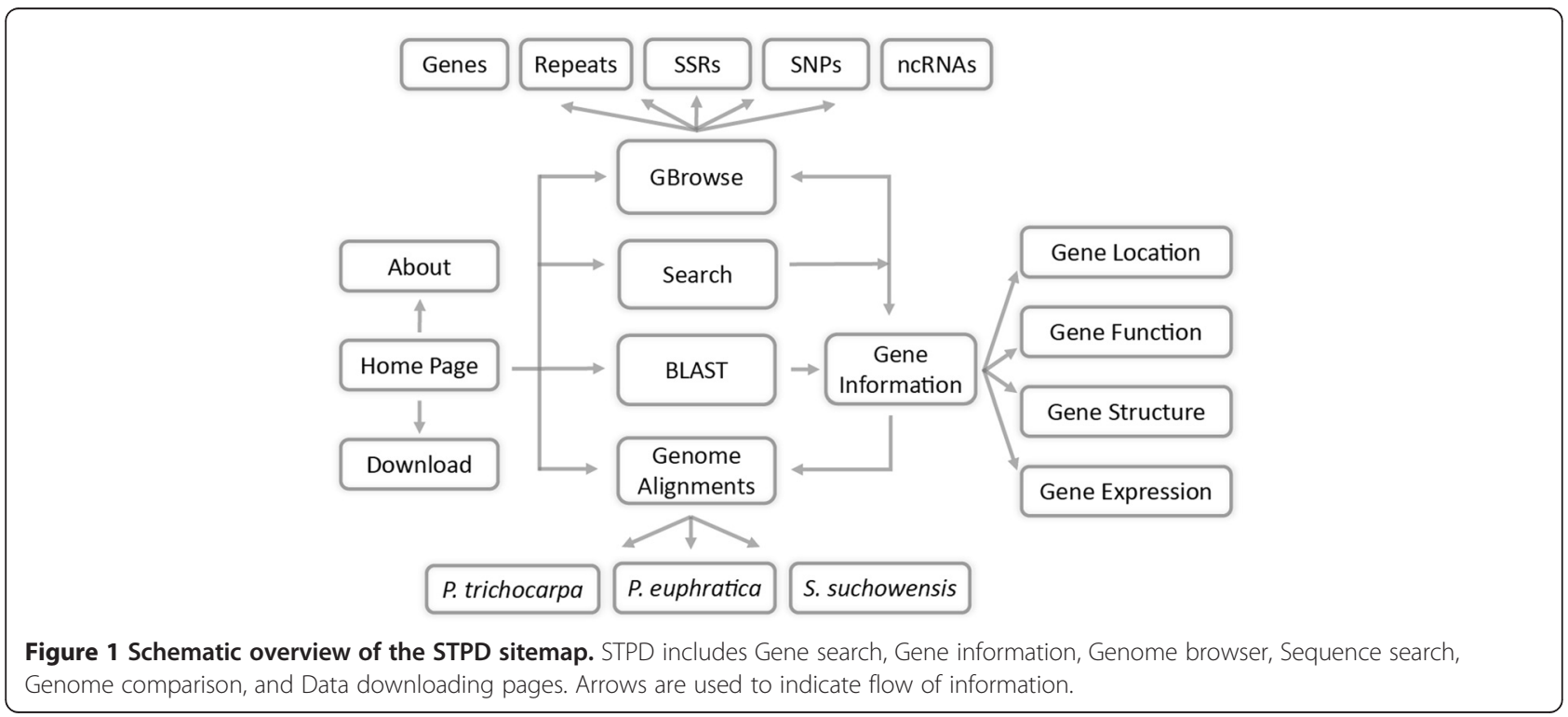


using HMMPfam, FPrintScan, ScanRegExp, ProfileScan, BlastProDom and HMMsmart with the following parameters: -format raw -goterms -iprlookup [14]. Gene Ontology information was then extracted from the InterProScan results with in-house Perl scripts.

\section{Comparative genomics}

Willows (Salix) share a common 'Salicoid' wholegenome duplication events with poplars, and their chromosomal structures are highly similar [15]. To better understand the salt-tolerant adaptive evolution, we performed a whole-genome comparison between $P$. euphratica, Salix Suchowensis and P. trichocarpa, a saltsensitive model plant with a high-quality reference genome [16], using Mercator and MAVID [17].

\section{Gene expression}

The STPD also includes gene expression data of $P$. euphratica calluses in response to salt stress together with those of $P$. tomentosa (a salt-sensitive poplar). The analysis was conducted on salt-stressed and non-stressed samples $(200 \mathrm{mM} \mathrm{NaCl}$ for $6,12,24$ and $48 \mathrm{~h}$ ), which generated from the same calluses used in genome sequencing [12].

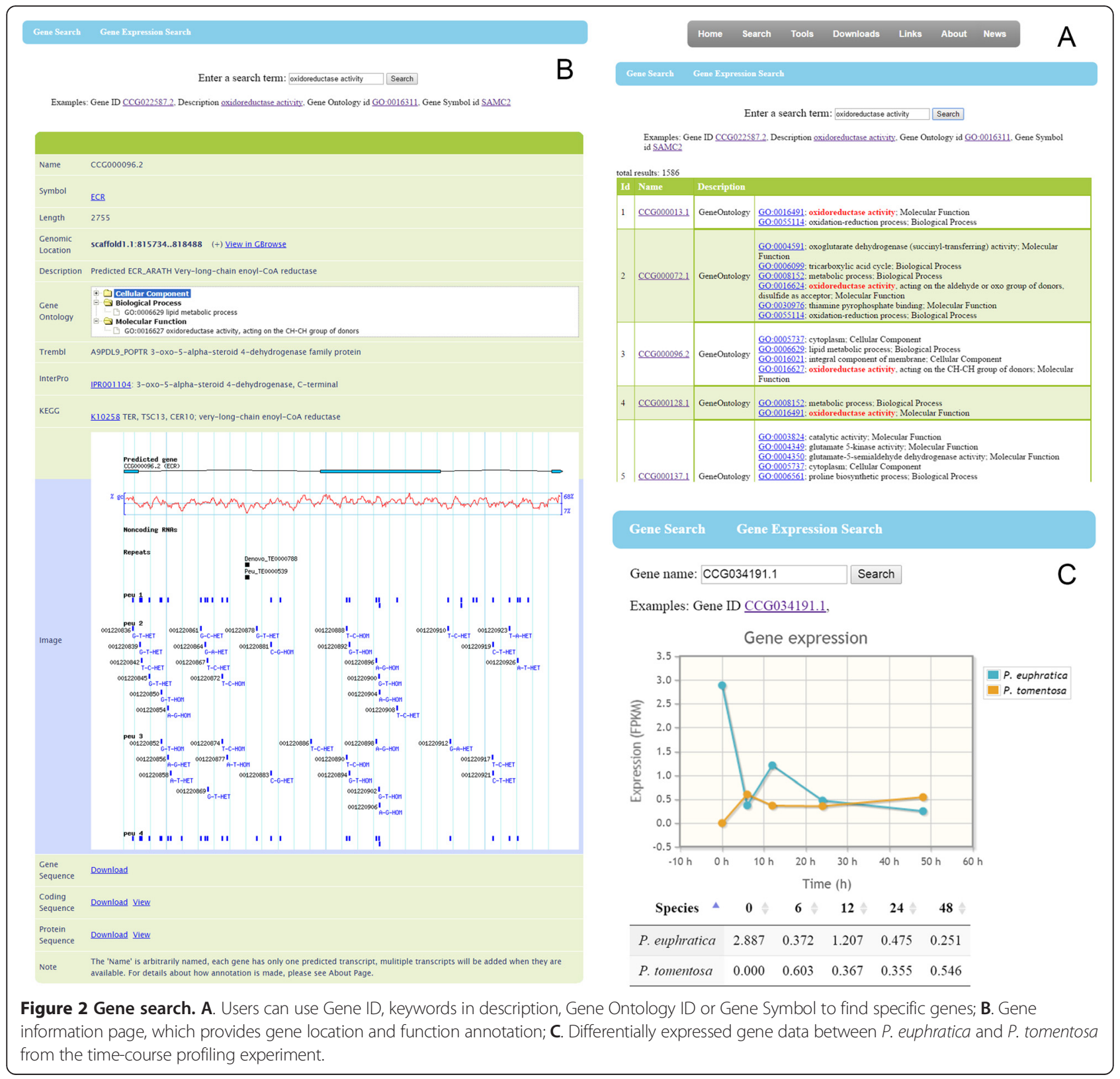




\section{Population genomics}

Whole-genome sequence data from four P. euphratica individuals located in China were integrated into the STPD. We aligned the reads to reference genome with BurrowsWheeler Aligner (BWA) [18], filtered duplicated reads using samtools [19], and realigned INDELs with GATK [20]. Finally we called single nucleotide polymorphisms (SNPs) with samtools mpileup, and dropped low quality SNPs (Quality <30). In total, 5,894,808 SNPs were identified. The average alignment coverage is $9 \mathrm{X}$ per individual.

\section{Utility}

\section{Gene search}

The keyword search is a very useful function, which was developed to identify genes based on their names or annotations, such as "MYB" or "Betaine aldehyde dehydrogenase". The detailed information about genes can be viewed by clicking on the gene's name or annotated identifier which links to relevant external website (Figure 2A). In the gene information page, users can view the annotated function, jump to GBrowse and download/view gene data (Figure 2B). This page also provides an overview of the gene's structure, genomic location and other related information. These annotations will be refreshed when gene models are updated. In the gene expression search page, the differentially expressed gene data between $P$. euphratica and $P$. tomentosa from the time-course profiling experiment is exhibited intuitively (Figure 2C).

\section{Genome browsing}

The Generic Genome Browser (GBrowse; http://gmod. org/wiki/GBrowse) was used to visualize the genome of the $P$. euphratica [21]. Users can browse genes, repetitive elements, non-coding RNAs, SSRs and SNPs in different tracks, and zoom into any region of interest in the

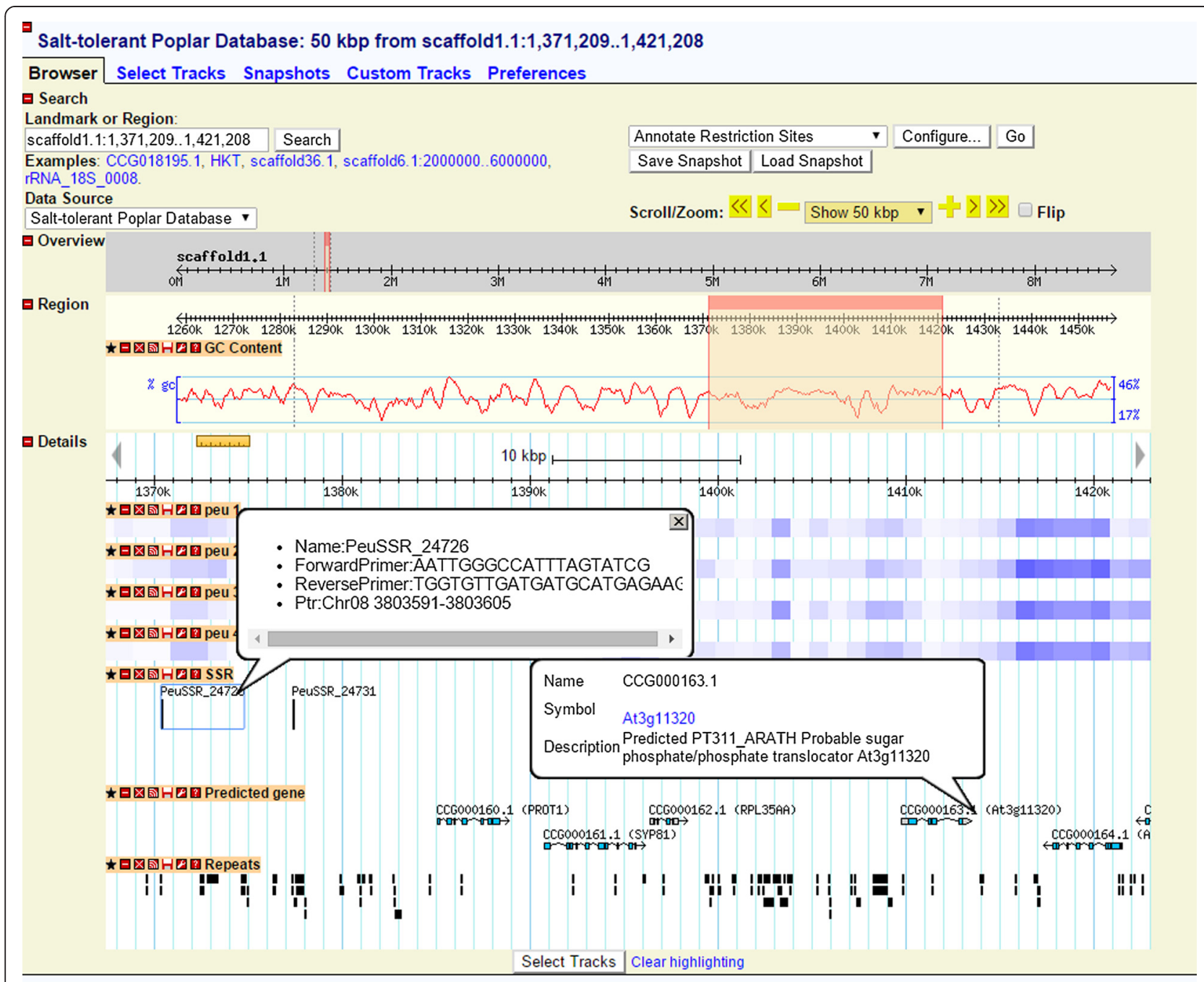

Figure 3 Browsing genome using GBrowse. Gene function description can be viewed by hovering the cursor over the gene name. When users click on a SSR name, a popup balloon will show the primer information and corresponding location in the P. trichocarpa genome. 
genome (Figure 3). By default, the repeats and SNPs are shown in density graph with darker color representing higher density. If the genome region is zoomed into certain ranges, summary mode will be turned off and detailed information of repeats and SNPs will be shown. When user hovers over a predicted gene or repeat element, a popup balloon will appear and show gene symbol, functional information or repeats family. By clicking on a SSR name, users will get the primer information and corresponding position in the $P$. trichocarpa genome. Clicking on a specific gene will redirect users to the corresponding gene information page, and users can return to the GBrowse by clicking on the "Tools" button within the navigation bar at the top of the page.

\section{Sequence similarity search}

BLAST is one of the most useful tools for searching the STPD. It can be used to find similar sequences against scaffolds or genes within $P$. euphratica genome. In the page of blast searching result, each hit is linked to the GBrowse view of the sequence.

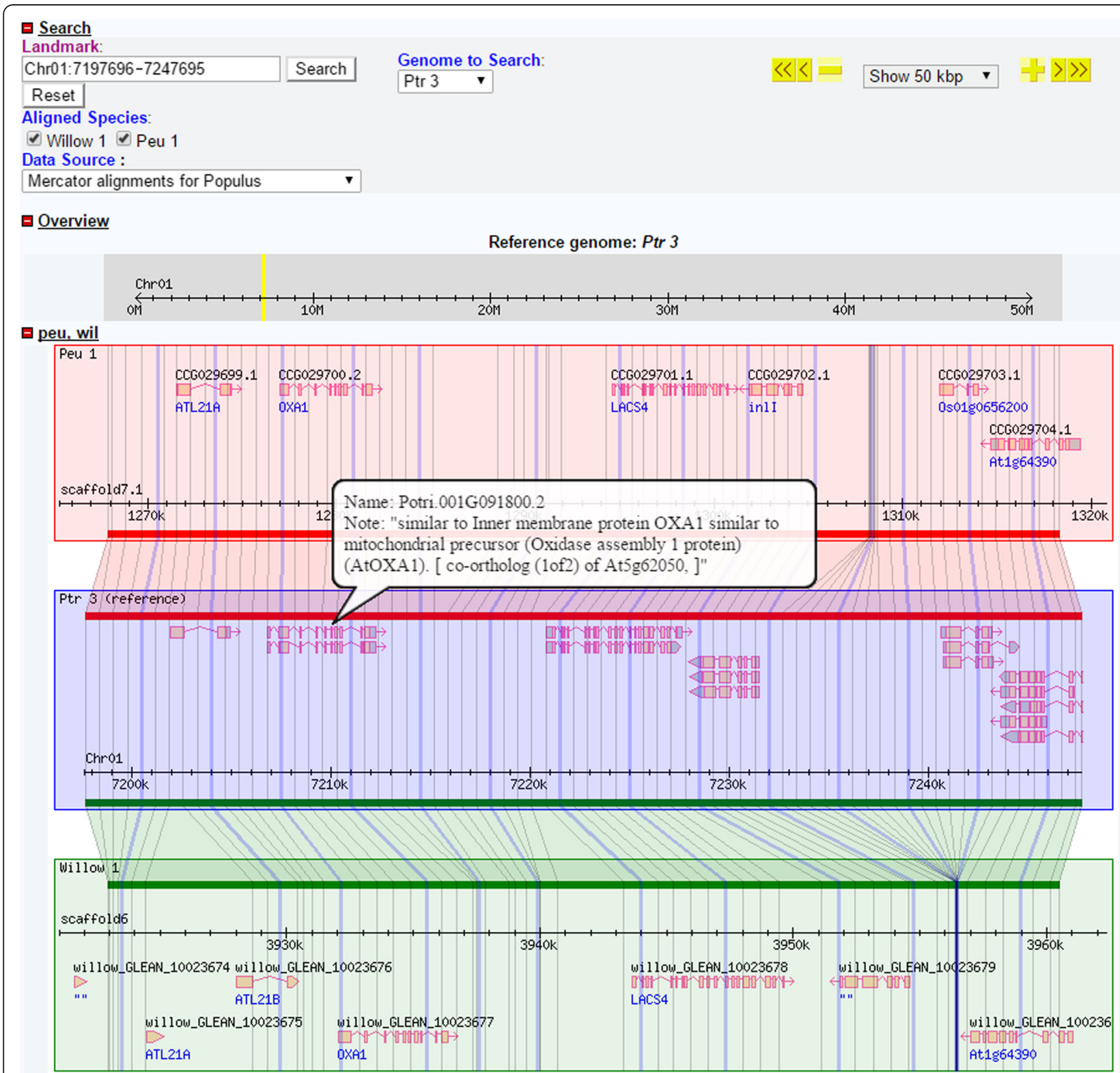

Figure 4 Genome alignments among $P$. euphratica, $P$. trichocarpa and S. suchowensis. Users can view any region of genome comparison by searching landmark or moving forward or backward using button in the right top corner. 


\section{Genome alignments browsing}

The genome alignments can be viewed by the Generic Synteny Browser (GBrowse-syn), which uses the same software framework as GBrowse [22]. Therefore, gene symbols and functional information will also be showed in a popup balloon when user hovers over a specific gene (Figure 4). Users can copy "Genomic Location" from the gene information page, paste it into "Landmark" textbox, select "Peu 1" in "Genome to Search", and then press search button to find the corresponding region in genome alignments.

\section{Data downloading}

Users can download the deposited sequences from the STPD to perform local searches against the whole genome or further academic studies. The MD5 checksum is also available for checking file integration.

\section{Discussion}

The STPD was created as a tool to facilitate the study of plant salt tolerance mechanism and poplar genomics. Compared with the existed poplar database (http://www. plantgdb.org/PtGDB/), our database provides access to a salinity tolerant poplar genome and its related genomic resources. It also offers comparative genomic data among $P$. euphratica, P. trichocarpa and S. suchowensis for researchers to better understand the adaptive evolutions of the closely related species. These data will promote the genetic dissection of stress-tolerant traits and accelerate the genetic improvement of cultivated poplars. The deposited sequences, related annotations and useful tools will allow users to find similar sequences in Populus, to browse genome features in the region of interest, and to retrieve data to further their interests in functional genomics, comparative genomics studies and molecular breeding. A number of research projects are currently in progress, including genome and transcriptome sequencing of Populus pruinosa, and genome re-sequencing of more than 200 individuals for $P$. euphratica, $P$. pruinosa and other related species. P. pruinosa is another salinity resistant poplar which is also distributed only in the desert regions of central Asia [23]. The genome-wide data getting from these studies will be integrated into the STPD as they become available.

\section{Conclusions}

We have developed the STPD to support studies on tree adaptation to salt stress and poplar genomics. The database provides genome-wide datasets of salinity tolerant Populus, along with data mining and visualization tools for studying the salt resistance in poplars. New datasets will be incorporated in the future in order to expand the scope and to help scientists dissect the salt tolerance in Populus thoroughly. The STPD offers a valuable repository for scientific community to better study plant salt tolerance, comparative genomics, extreme environment adaptation and molecular breeding.

\section{Availability and requirements}

Database name: STPD

Database homepage: http://me.lzu.edu.cn/stpd/

Browser requirement: JavaScript should be enabled; we recommend the use of the Chrome or Firefox web browsers for an optimal experience.

Datasets in the STPD are freely available for academic use. For all other uses, please contact Quanjun Hu, huquanjun@gmail.com.

\section{Abbreviations}

BLAST: Basic local alignment search tool; BWA: Burrows wheeler aligner; GATK: Genome analysis tool kit; GBrowse: Generic genome browser; GBrowse-syn: Generic synteny browser kaas, kegg automatic annotation server; KEGG: Kyoto encyclopedia of genes and genomes; SNP: Single nucleotide polymorphism; SSR: Simple sequence repeat; STPD: Salinity Tolerant Poplar Database; TE: Transposable element.

\section{Competing interests}

The authors declare that they have no competing interests.

\section{Authors' contributions}

QJH designed the project; YZM, TX, DSW constructed the database; SS tested the web applications and provided feedback. YZM, QJH, TX wrote the paper and TM, QJL revised the manuscript. All authors read and approved the final manuscript.

\section{Acknowledgements}

This work is supported by the National High Technology Research and Development Program of China (863 Program, No. 2013AA100605), National Key Project for Basic Research (2012CB114504), the Fundamental Research Funds for the Central Universities (Izujbky-2009-k05), the China Postdoctoral Science Foundation funded project (2014 M552354) and the 985 and 211

Projects of Lanzhou University.

Received: 12 November 2014 Accepted: 28 February 2015

Published online: 17 March 2015

References

1. Türkan I, Demiral T. Recent developments in understanding salinity tolerance. Environ Exp Bot. 2009;67:2-9.

2. Flowers TJ. Improving crop salt tolerance. J Exp Bot. 2004;55(396):307-19.

3. Janz D, Lautner S, Wildhagen $H$, Behnke K, Schnitzler J-P, Rennenberg H, et al. Salt stress induces the formation of a novel type of "pressure wood" in two Populus species. New Phytol. 2012;194:129-41.

4. Wu H-J, Zhang Z, Wang J-Y, Oh D-H, Dassanayake M, Liu B, et al. Insights into salt tolerance from the genome of Thellungiella salsuginea. Proc Natl Acad Sci. 2012;109:12219-24.

5. Jarvis DE, Ryu C-H, Beilstein MA, Schumaker KS. Distinct roles for SOS1 in the convergent evolution of salt tolerance in Eutrema salsugineum and Schrenkiella parvula. Mol Biol Evol. 2014;31(8):2094-107.

6. Eckenwalder JE. Systematics and evolution of Populus. In: Stettler RF, Brandshaw Jr HD, Heilman PE, Hinckley TM, editors. Biology of Populus and its implications for management and conservation. Ottawa: NRC Research Press; 1996. p. 7-32.

7. Chen S. Polle A. Salinity tolerance of Populus. Plant Biol. 2010;12:317-33.

8. Fischer U, Polle A. Populus responses to abiotic stress. In: Jansson S, Bhalerao R, Groover A, editors. Genetics and genomics of Populus. New York: Springer; 2010. p. 225-46.

9. Jansson S, Douglas CJ. Populus: a model system for plant biology. Annu Rev Plant Biol. 2007;58:435-58. 
10. Chen YP, Chen YN, Li WH, Xu CC. Characterization of photosynthesis of Populus euphratica grown in the arid region. Photosynthetica. 2006:44:622-6.

11. Ottow EA, Brinker M, Teichmann T, Fritz E, Kaiser W, Brosché M, et al. Populus euphratica displays apoplastic sodium accumulation, osmotic adjustment by decreases in calcium and soluble carbohydrates, and develops leaf succulence under salt stress. Plant Physiol. 2005;139:1762-72.

12. Ma T, Wang J, Zhou G, Yue Z, Hu Q, Chen Y, et al. Genomic insights into salt adaptation in a desert poplar. Nat Commun. 2013;4:2797.

13. Moriya Y, Itoh M, Okuda S, Yoshizawa AC, Kanehisa M. KAAS: an automatic genome annotation and pathway reconstruction server. Nucleic Acids Res. 2007;35 suppl 2:W182-5.

14. Jones P, Binns D, Chang H-Y, Fraser M, Li W, McAnulla C, et al. InterProScan 5: genome-scale protein function classification. Bioinformatics. 2014;30(9):1236-40.

15. Dai X, Hu Q, Cai Q, Feng K, Ye N, Tuskan GA, et al. The willow genome and divergent evolution from poplar after the common genome duplication. Cell Res. 2014;24:1274-7.

16. Tuskan GA, DiFazio S, Jansson S, Bohlmann J, Grigoriev I, Hellsten U, et al. The Genome of Black Cottonwood, Populus trichocarpa (Torr. \& Gray). Science. 2006;313(5793):1596-604.

17. Dewey CN. Aligning multiple whole genomes with Mercator and MAVID. Methods Mol Biol. 2007;395:221-36.

18. Li H, Durbin R. Fast and accurate short read alignment with Burrows-Wheeler transform. Bioinformatics. 2009;25(14):1754-60.

19. Li H. A statistical framework for SNP calling, mutation discovery, association mapping and population genetical parameter estimation from sequencing data. Bioinformatics. 2011;27(21):2987-93.

20. DePristo MA, Banks E, Poplin R, Garimella KV, Maguire JR, Hartl C, et al. A framework for variation discovery and genotyping using next-generation DNA sequencing data. Nat Genet. 2011;43:491-8.

21. Stein LD, Mungall C, Shu S, Caudy M, Mangone M, Day A, et al. The generic genome browser: a building block for a model organism system database. Genome Res. 2002;12:1599-610.

22. McKay SJ, Vergara IA, Stajich JE. Using the generic synteny browser (GBrowse_syn). Curr Protoc Bioinformatics. 2010;31:9.12.1-25.

23. Zhang J, Feng J, Lu J, Yang Y, Zhang X, Wan D, et al. Transcriptome differences between two sister desert poplar species under salt stress. BMC Genomics. 2014;15:337.

\section{Submit your next manuscript to BioMed Central and take full advantage of:}

- Convenient online submission

- Thorough peer review

- No space constraints or color figure charges

- Immediate publication on acceptance

- Inclusion in PubMed, CAS, Scopus and Google Scholar

- Research which is freely available for redistribution 